\title{
El estrés innecesario durante el desarrollo de un trabajo de investigación
}

\author{
Un necessary stress during the development of a research project \\ Yercin Mamani Ortiz ${ }^{1}$
}

\section{Señores: Gaceta Médica Boliviana}

Considerando que su revista es de difusión masiva en la comunidad científica local, aprovecho esta vía para poder llegar a los nuevos investigadores en salud, para motivarlos y plantearles algunas recomendaciones que les permitan evitar el estrés innecesario que se genera durante todo proceso de investigación. El nivel de estrés y su expresión biológica, psicológica u otra, dependerá de la capacidad de resiliencia que desarrolla cada investigador durante su formación y/o conducción de un trabajo de investigación, en cualquiera de los diferentes tipos de investigación.

Todos los seres humanos tenemos instintos y tendencia a cometer errores o sesgos durante el proceso de comprensión de nuestra realidad; esto no está exento para los investigadores, siendo en algunos casos innatos y en otros, derivados de nuestra exposición a corrientes ideológicas de nuestros profesores y tutores, o también a las tendencias internacionales que muchas veces marcan las líneas para el desarrollo de investigaciones.

El profesor Hans Rosling y colaboradores, en su libro "Factfulness : Ten reasons we're wrong about the world and why things are better than you think"1 (Una realidad: Diez razones por las que estamos equivocados con respecto al mundo y por qué las cosas son mejores de lo que piensa), nos hablan sobre cómo podemos reemplazar nuestra cosmovisión dramática por una cosmovisión basada en la evidencia; de esta manera mejorar nuestra toma de decisiones, estar preparados y en alerta frente a los peligros o posibilidades reales de fracaso, de manera que evitemos estar constantemente estresados por las cosas incorrectas. Por esta razón, el gobierno sueco lo incluye como una de las lecturas obligatorias para los estudiantes de nivel secundario.

A continuación, planteo una homologación de estas pautas para tomar el control sobre nuestros instintos innatos o desarrollado frente al desarrollo de procesos de investigación, de manera que podamos reconocerlos y evitar el estrés innecesario:

1) El instinto de la diferencia absoluta: ningún conocimiento científico es absoluto, tampoco lo será aquel que generemos con nuestro trabajo de investigación. Mientras más profundo sea nuestro análisis, entenderemos mejor nuestro objeto de estudio, y las diferencias entre modelos, teorías y conceptos vigentes. Un ejemplo clásico sobre este punto es el referido al efecto de un factor de riesgo sobre un evento de salud, que puede ser completamente diferente entre ${ }^{2}$ individuos o grupos poblacionales. Por lo tanto, no te sientas mal, si tus resultados no llegan a un $100 \%$ de solidez, validez o correlación clínica, esto es parte de todos los procesos de investigación.

2) El instinto de "Positivización": no te frustres innecesariamente frente a hallazgos negativos o contradictorios en tu trabajo de investigación. En la comunidad académica y científica es común enfatizar los resultados positivos, y en muchos casos se trata de evitar comunicar los resultados negativos o contradictorios de un estudio, tanto por los autores, como algunas revistas de alto impacto. Sin embargo, un resultado negativo también significa un aporte a la evidencia científica, pues demuestra que, en un contexto particular, los efectos esperados sobre un fenómeno pueden ser parcial o completamente diferentes o contradictorios a los hallados por otros equipos de investigación ${ }^{2}$.

3) El instinto de línea recta o fija: el desarrollo de una patología o evento en salud no siempre es predecible mediante modelos estadísticos. Muchas veces este fenómeno puede ser cíclico o sinusoidal; tal como ocurre con las enfermedades infecto-contagiosas consideradas eliminadas o erradicadas, que muchas

\footnotetext{
${ }^{1}$ Médico Cirujano, Investigador en IIBISMED-U.M.S.S., Doctorante en Salud Pública

en Universidad de Umeå-Suecia.

${ }^{*}$ Correspondencia a:Yercin Mamani Ortiz

Correo electrónico: yercin2003@hotmail.com

Recibido el 10 de septiembre de 2018. Aceptado el 23 de octubre de 2018
} 
veces generan nuevos brotes epidemiológicos que no pueden ser pronosticados con antelación. Así como el curso clínico de un paciente, ya sea parte o no de un trabajo de investigación, generando datos extremos (muy elevados o muy bajos en comparación a la media), esto no debe desalentarnos, pues existen métodos que permiten corregir los mismos o en su defecto, siempre queda la posibilidad de excluirlos del estudio.

4) El instinto de miedo: existen un mayor número de trabajos que son excluidos para su publicación por faltas éticas que por errores metodológicos o estadísticos. Muchas veces magnificamos las limitaciones de nuestro estudio y asumimos que anulan nuestros hallazgos. Un ejemplo clásico es el conflicto con el tipo de diseño metodológico, que puede resolverse simplemente con una adecuada clasificación y redacción; o el conflicto por el tamaño muestral reducido que limita la significancia estadística del estudio, pero no invalida nuestros hallazgos y finalmente el conflicto por una distribución de datos que no es normal, que puede solucionarse utilizando métodos de estadística no paramétrica durante el análisis. Por el contrario, no se enfatiza en el componente ético en la fase del diseño, implementación o análisis de la información, que en cualquiera de los casos pueden limitar o anular la publicación de nuestra investigación en una revista científica indexada, en la mayoría de las revistas, la aprobación de un comité de ética es indispensable ${ }^{3}$.

5) El instinto del tamaño: comprender la diferencia entre tasa, proporción y porcentaje, puede evitarnos muchos conflictos a la hora de interpretar nuestros resultados. Un porcentaje pequeño no siempre implica un efecto pequeño, a su vez una proporción elevada no necesariamente implica un alto número de casos. Muchas veces se genera un conflicto innecesario cuando se utiliza frecuencias absolutas (números absolutos) en lugar de las relativas (porcentajes), se sugiere utilizar frecuentas relativas para la presentación de los resultados, siempre y cuando el tamaño muestral no sea demasiado pequeño, en cuyo caso debe utilizarse números absolutos; de esta manera evitaremos una preocupación innecesaria sobre la magnitud de nuestros hallazgos.

6) El instinto de generalización: toda investigación científica debe evitar los estereotipos y generalizar demasiado nos puede llevar a cometer la falacia ecológica ${ }^{4}$. Es común para algunos investigadores, suponer que todos los miembros de un grupo de estudio tienen en común los mismos rasgos que definen al grupo, o asumen que la magnitud de los efectos de un factor determinados en un estudio, se reproducen de manera similar en todos los individuos. Debemos ser conscientes de que, si bien nuestras investigaciones tienen por objetivo obtener resultados generalizables a la población, esto no implica que encontremos el mismo resultado en todos los sujetos; de manera que evitemos conflictos innecesarios durante el análisis e interpretación de nuestros resultados.

7) El instinto del destino: el método científico o la estructura de presentación de una investigación no es dogmática, esa perspectiva es completamente contradictoria a las bases que sustentan la investigación científica: la refutabilidad. La ciencia tiene que ser falible, esta es la base del progreso científico. En este sentido, muchos diseños metodológicos se van modificando durante su implementación, al igual que el tipo de análisis estadístico presentado en el plan inicial; de ahí la importancia de las pruebas piloto o de validación de los instrumentos; esto nos evitará percances innecesarios a la hora de implementar nuestro estudio. Otro ejemplo clásico es el formato de presentación del trabajo de investigación; cada institución académica y cada revista científica tiene sus propias normas en la que establecen la estructura de presentación de los trabajos de investigación. Revisar estas normas con antelación, nos evitará conflictos innecesarios a la hora de presentar el documento final o en su defecto que sea rechazado por razones de formato.

8) El instinto de la perspectiva única: nadie investiga lo que no conoce y nadie conoce todo. Esta relación dialéctica que se genera en los procesos de investigación fue la base para la generación de equipos multidisciplinarios para el desarrollo de procesos de investigación y en la actualidad es la recomendación más importante de los organismos financiadores. Este tipo de equipos nos permite generar una perspectiva más amplia sobre temas que están fuera de nuestra área de formación y evitar un sesgo innecesario en la recolección de información, análisis e interpretación de nuestros hallazgos. En nuestro medio, hemos sugerido en varias instancias que los trabajos de tesis de pre y postgrado deberían ser supervisadas por un asesor técnico (especialista en el objeto de estudio), con el apoyo de uno metodológico y/o bioestadístico. Nuestra experiencia nos muestra que la conformación de este equipo facilita el desarrollo de un trabajo de investigación y enriquece el análisis de los resultados obtenidos, mejorando su calidad en procura de su publicación.

9) El instinto de culpa: el investigador principal no es el único culpable y no se puede controlar todos los factores. Es común culpabilizarnos ante resultados que no son los esperados para confirmar o refutar completamente nuestra hipótesis, muchas veces existen factores externos a nuestra investigación y que no están incluidas entre las variables evaluadas. Eso no quiere decir que el trabajo sea inválido, simplemente que se requiere realizar más estudios que exploren aquellos factores o variables no consideradas en primera instancia y que no eran parte de los objetivos de estudio. Los resultados de una tesis deben responder únicamente a los objetivos planteados en el estudio; todo aquello que esté fuera de los mismos, es información complementaria; por ello la importancia de contar con un buen protocolo que es aprobado por todo el equipo de investigación que tiene una responsabilidad administrativa y legal sobre el documento final, además de 
los comités institucionales de investigación y/o de ética que la avalan.

10) El instinto de urgencia: lo más rápido no siempre es lo mejor, no dejes todo para el último momento. Una de las mayores preocupaciones en el desarrollo de un trabajo de investigación son los plazos de entrega del documento final. Muchos tesistas pretenden culminar su trabajo en menos de un semestre, que si bien puede ser factible (dependiendo del área de investigación), no siempre es lo más recomendado, así como tampoco el que extienda por mucho tiempo; pues genera cansancio, estrés, frustración y en muchos casos el abandono del proyecto. No te acumules todo para el último día del plazo de entrega, plantearse metas cortas (por ejemplo: un capítulo de tesis por semana) puede reducirte el estrés innecesario. La mayor cantidad de errores ocurren por la premura de cumplir con los plazos fatales de presentación del documento final.

Seguramente muchos se autoidentificarán con alguno de los puntos tratados, y estoy seguro de que existen muchos otros que no fueron tratados. Confió en que estas pautas sean recibidas favorablemente, y que cada investigador (en especial aquel tesistas de pre y postgrado) pueda hacer una introspección para mejorar su capacidad de resiliencia y finalizar con una experiencia positiva en el desarrollo de su investigación.

Palabras clave: estrés, tesis, resiliencia, investigación en salud.

\section{Referencias bibliográficas}

1. U.S. National Library of Medicine. Number of Authors per MEDLINE/PubMed Citation [Internet]. [citado 24 de mayo de 2018]. Disponible en: https://www.nlm.nih.gov/bsd/authors1.html

2. Smith R. The trouble with medical journals. Reimpr. Boca Raton: CRC press; 2011. 292 p.

3. Carot G, Vallés X, Bonmatí M. El conflicto de intereses en los profesionales de la salud. Bioética \& Debat. 2017;23(81):2-25.

4. Fugh-Berman A, McDonald CP, Bell AM, Bethards EC, Scialli AR. Promotional tone in reviews of menopausal hormone therapy after the Women's Health Initiative: an analysis of published articles. PLoS Medicine. 15 de marzo de 2011; 8(3):e1000425.

5. ICMJE. Recommendations for the Conduct, Reporting, Editing, and Publication of Scholarly Work in Medical Journals [Internet]. ICMJE; 2017 [citado 24 de mayo de 2018]. Disponible en: http:// www.icmje.org/icmje-recommendations.pdf 